\title{
Model Point-of-Care Ultrasound Curriculum in an Intensive Care Unit Fellowship Program and Its Impact on Patient Management
}

\author{
Keith Killu, ${ }^{1}$ Victor Coba, ${ }^{1}$ Michael Mendez, ${ }^{2}$ Subhash Reddy, ${ }^{3}$ Tanja Adrzejewski, \\ Yung Huang, ${ }^{5}$ Jessica Ede, $^{6}$ and Mathilda Horst ${ }^{1}$ \\ ${ }^{1}$ Department of Surgery/Division of Trauma and Critical Care, Henry Ford Hospital, 2799 W. Grand Boulevard, \\ Detroit, MI 48202, USA \\ ${ }^{2}$ Department of Pulmonary and Critical Care, Henry Ford Hospital, Detroit, MI 48202, USA \\ ${ }^{3}$ Cleveland Clinic, Cleveland, OH 44195, USA \\ ${ }^{4}$ Beaumont Hospital, Royal Oaks, MI 48073, USA \\ ${ }^{5}$ Doctors Medical Center, Modesto, CA 95350, USA \\ ${ }^{6}$ Wayne State University, Detroit, MI 48202, USA \\ Correspondence should be addressed to Keith Killu; kkillu1@hfhs.org
}

Received 10 August 2014; Revised 2 October 2014; Accepted 30 October 2014; Published 16 November 2014

Academic Editor: Roland M. Schein

Copyright ( $\odot 2014$ Keith Killu et al. This is an open access article distributed under the Creative Commons Attribution License, which permits unrestricted use, distribution, and reproduction in any medium, provided the original work is properly cited.

Objectives. This study was designed to assess the clinical applicability of a Point-of-Care (POC) ultrasound curriculum into an intensive care unit (ICU) fellowship program and its impact on patient care. Methods. A POC ultrasound curriculum for the surgical ICU (SICU) fellowship was designed and implemented in an urban, academic tertiary care center. It included 30 hours of didactics and hands-on training on models. Minimum requirement for each ICU fellow was to perform 25-50 exams on respective systems or organs for a total not less than 125 studies on ICU. The ICU fellows implemented the POC ultrasound curriculum into their daily practice in managing ICU patients, under supervision from ICU staff physicians, who were instructors in POC ultrasound. Impact on patient care including finding a new diagnosis or change in patient management was reviewed over a period of one academic year. Results. 873 POC ultrasound studies in 203 patients admitted to the surgical ICU were reviewed for analysis. All studies included were done through the POC ultrasound curriculum training. The most common exams performed were 379 lung/pleural exams, 239 focused echocardiography and hemodynamic exams, and 237 abdominal exams. New diagnosis was found in $65.52 \%$ of cases $(95 \%$ CI $0.590,0.720)$. Changes in patient management were found in $36.95 \%$ of cases (95\% CI 0.303, 0.435). Conclusions. Implementation of POC ultrasound in the ICU with a structured fellowship curriculum was associated with an increase in new diagnosis in about $2 / 3$ and change in management in over $1 / 3$ of ICU patients studied.

\section{Introduction}

Ultrasound has been used since the 1940s to aid in patient management and radiologists have long appreciated its benefits. POC ultrasound is defined as ultrasonography brought to the patient and performed by the provider in real time [1]. The emergence of POC ultrasound was over 20 years ago and the initial advocates were tertiary centers applying this technology to assist the clinicians in patient management [2, 3]. Given the advantages of availability and the reproducible results, POC ultrasound became a useful tool in the clinician armamentarium for managing their patients.

POC ultrasound is becoming an integral part of many ICUs, serving as a bedside tool to assist the ICU physician in patient management. In the last two decades a more focused approach to bedside ultrasound has emerged and its use has 
expanded in assessing trauma patients $[2,3]$, hemodynamics $[4-6]$, and disaster incidences $[7,8]$ as well as, many applications by the Emergency Medicine (EM) physicians across the globe [9-11], applications for procedures [10], and even found its applications in space $[12,13]$.

Teaching nonradiology residents, fellows, and clinicians about the use and implementation of POC ultrasound has been integrated in many academic and nonacademic centers and is currently being taught as part of the curriculum in medical schools $[14,15]$, in residency programs $[16,17]$, and through remote guidance to rural areas $[18,19]$ to individuals who are naïve to the technology.

Currently, POC ultrasound applications and training in the ICU $[17,20]$ are expanding rapidly, yet, only limited model curriculums exist for teaching and implementing POC ultrasound in the ICU, and their impact on patient care is not clear [21], and currently ultrasound training is highly recommended but not mandated by the Accreditation Council for Graduate Medical Education (ACGME) for critical care training. Adequate teaching and competency are needed to ensure patient safety; otherwise consequences of applying inadequate knowledge [22] are unknown. The Society of Critical Care Medicine and the American College of Chest Physicians offer courses for training and certification of completion of courses as well as certification of training.

Most studies have focused on the aspects of a steep learning curve $[23,24]$, organ or system applications, and the nonradiologist performance of specific ultrasound examination as the Focused Assessment with Sonography for Trauma (FAST) [2, 3], echocardiography [23, 24], lung exams [25], and others, as well as expanding literature emphasizing the benefits of using bedside ultrasound during procedures $[10$, $26,27]$ and hemodynamic support [28].

The gaps in formal training programs have been identified by the American College of Chest Physicians [29] and The American Society of Echocardiography and American College of Emergency Physicians addressed in separate published consensus statements and reviews [30].

\section{Materials and Methods}

After approval by the institutional review board, a retrospective review of POC ultrasound studies and patients charts was conducted. A POC ultrasound curriculum for the surgical ICU (SICU) fellowship was designed and implemented in an urban, academic tertiary care center. As many other tertiary educational centers, we started implementing POC ultrasound in our surgical ICU where the focus was to aid in patient management and in the process and teach our surgical ICU fellows the applications of POCUS. During this process we realized the need for developing a structured curriculum for teaching. A needs assessment evaluation for a structured POC ultrasound curriculum was conducted through interviews and questionnaires to our ICU faculty and fellows, as well as conducting literature searches [16-21, 2325]. The POC ultrasound curriculum developed in our ICU was adopted from the EM model [30] with modifications to suit the needs of patients and treating physicians in the ICU settings.
After the initial training period of the ICU fellows, they started implementing POCUS in their daily practice and management of their patients. All ultrasound exams performed were documented and reviewed by an ICU attending who is experienced in POC ultrasound, and certified by the American Registry for Diagnostic Medical Sonography (ARDMS). Data was stored as part of the patient profile and management and also as a record to evaluate the fellow's performances.

After approval by the institutional review board, a retrospective review of POC ultrasound studies and patients charts was conducted. POC ultrasound was performed by the ICU fellows and was part of the patient management and the standard of care in many institutions.

2.1. The Curriculum. The curriculum model was adopted from the American College of Emergency Physicians/American Society of Echocardiography (ACEP/ASE) model [30] with modifications. There is a great deal of overlap between the EM and ICU regarding the use of POC ultrasound, and our goal was to create a curriculum that is similar to a successful model which has been in place for a period of time with modifications and adjustments that are suitable for the critical care settings. The elements of the curriculum were divided into system or organ based sessions. The curriculum elements included mainly the following:

(1) physics and knobology;

(2) lung and pleural ultrasonography;

(3) abdominal ultrasonography including gallbladder, liver, spleen, kidneys, aorta, FAST, and E-FAST;

(4) vascular procedures: guidance for vascular access;

(5) basic critical care echocardiography (CCE);

(6) hemodynamic assessment including limited echocardiography, lungs, inferior vena cava, and internal jugular vein.

The modes of instructions included session format of 30 minutes of didactics followed by 60 minutes of hands-on sessions on models to reinforce the techniques. A total of 12 sessions were conducted every year at the beginning of the academic year for the new fellows. The fellows were required to review the ultrasound topic for the organ or system or the protocol to be discussed by referring to a handbook, textbook, and articles on POC ultrasound. The teaching sessions were provided by ICU and EM attending physicians, cardiologists, radiologists, and cardiac technicians.

Emphasis was placed on certain aspects of POC ultrasound. These aspects were as follows:

(1) resuscitative: use of POC ultrasound in acute resuscitation;

(2) diagnostic: use of POC ultrasound as an aid in diagnosis;

(3) symptom or sign-based: use of POC ultrasound in an algorithm or protocol adopted from literature;

(4) procedure guidance: use of POC ultrasound as an aid to guide a procedure; 
TABLE 1: POC ultrasound curriculum exams requirements. To be performed by each ICU fellow during their fellowship.

\begin{tabular}{lc}
\hline Ultrasound exam element & Reviewed exams \\
\hline Lung and pleura & $25-50$ \\
Abdominal (including FAST) & $25-50$ \\
Vascular access (and other procedures) and DVT & $25-50$ \\
Basic echocardiography & $25-50$ \\
Hemodynamic evaluation & $25-50$ \\
\hline
\end{tabular}

(5) therapeutic and monitoring: use of POC ultrasound in therapeutics or in physiological monitoring.

Minimum requirement for each ICU fellow was to perform 25-50 exams on respective systems or organs for a total not less than 125 exams on ICU patients during their fellowship. The requirements for achieving competency in different elements are listed in Table 1 . The competency requirements were adopted from the EM curriculum [30] that has been in use for many years. Part of the total exams required by each fellow was directly supervised by the attending ICU physician before the fellow is deemed able to perform POCUS and submit reports for review. This decision was made taking into consideration other societies recommendation [30] and after direct observation of the studies performed by the ICU fellows.

Some applications, like the procedural vascular access, require fewer cases, given the prior knowledge and clinical experience with the landmark guided techniques. Documentation was done for all POC ultrasound exams and stored initially in the ultrasound unit, or on a worksheet, and some were transferred to a server system (Qpath) (Telexy Healthcare, Everett, WA, USA) that was housed in our institution where the exam video clips, still images, and reports could be stored.

Initially, the exams were supervised by one of the instructors (a total of 3 instructors performed the supervision) as shown in Table 1, after which the fellows (total of 3 fellows for the academic year) were deemed able to perform the exams on their own but still needed review by the attending physician for the final diagnosis to be confirmed.

Competency evaluations and quality assurance (QA) systems were an integral part of the curriculum. The objective of the QA process is to evaluate the images for technical competence and interpretations for clinical accuracy and to provide feedback to improve physician performance. The methods for QA included the following.

(i) Direct supervision of the fellow performance of POC ultrasound exam by an expert ICU physician: it is an ideal form of QA and practice performance activities. Parameters evaluated included image resolution, anatomic definition, and other image quality acquisition aspects such as gain, depth, orientation, and focus. Also the fellow's competency in POCUS was evaluated in image interpretation and forming a diagnosis. (ii) Providing feedback to the fellow after reviewing their POC ultrasound examination: the review and feedback were done in person and included the review of video clips and reports or commenting on reports and images that were obtained and stored. Reviewers evaluated images for accuracy and technical quality and communicated the reviews to the ICU fellow.

2.2. Data Collection. Data was reviewed for 203 consecutive patients (a total of 873 ultrasound exams) admitted to the SICU over a one-year period where POC ultrasound was performed as part of their management. The admitting diagnosis was made by the attending ICU physician and was established on the basis of history, physical examination, laboratory, and radiological findings. POC ultrasound was performed to aid in patient diagnosis and management. All evaluations were done almost in real time by the fellows or within minutes of the exam to ensure correct diagnosis and aid in patient management. Review of the reports was done by more than one attending physician to confirm the ultrasound diagnosis, and where there was discrepancy, the reports were not included in the final analysis. The reviewing physician was not blinded to the patient diagnosis. Outcomes measured were whether POC ultrasound led to finding of at least one new diagnosis not identified without the use of POC ultrasound and whether POC ultrasound resulted in change in management of the patient (defined as any change in medications, fluids, new laboratory or radiological tests, or new procedures).

A retrospective analysis of the results was done using Statistical Product and Service Solutions software (SPSS). The statistical methodology included retrospective descriptive frequencies and percentages of the various study types that were obtained. The two outcomes of interest (new diagnosis and change in management) were recorded as yes or no based on if they occurred in at least one of the ultrasound studies completed on each patient. The resulting overall percentages and corresponding 95\% confidence intervals (CI) were calculated for the two outcomes.

\section{Results}

A POC ultrasound curriculum was developed and implemented in the SICU fellowship program. Minimum requirement for each ICU fellow was to perform 25-50 exams on respective systems or organs for a total not less than 125 exams on ICU patients during their fellowship.

During a one-year academic period, 3 ICU fellows performed 873 ultrasound studies in 203 consecutive patients, and the data was included for analysis in a retrospective clinical investigation. The ultrasound exams performed for procedural purposes were not included in the analysis.

The most common exams performed were 379 (43.41\%) lung and pleura exams, 239 cardiovascular exams (including the limited echocardiography and hemodynamic assessment) (27.37\%), and 237 abdominal exams (including FAST, gallbladder, general abdomen, and pelvis) (27.14\%) (Figure 1). 


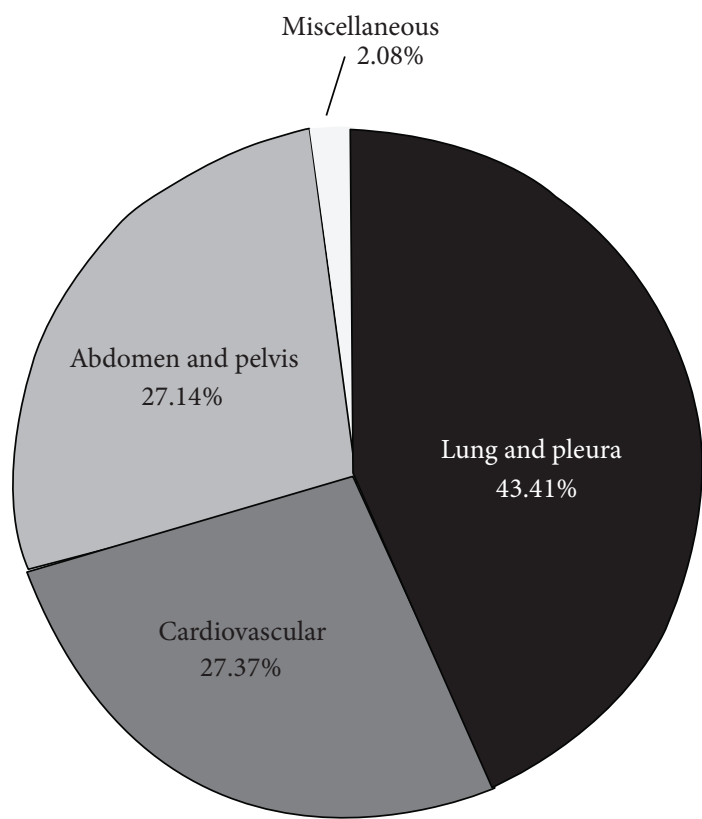

FIgURE 1: POCUS exams performed by the ICU fellows during a one-year academic period.

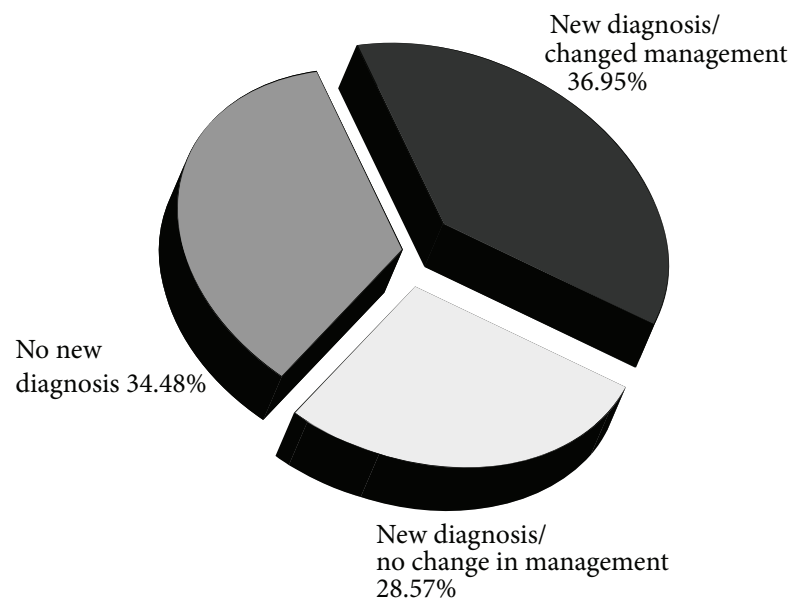

Figure 2: By implementing the Point-of-Care ultrasound curriculum, a new diagnosis is found in $65.52 \%$ of patients and a change in patient management was found in $36.95 \%$ of patients studied. $95 \%$ CI $(0.303,0.435)$.

The POC ultrasound examinations resulted in at least 1 new diagnosis in $65.52 \%$ of patients, $95 \%$ CI $(0.590,0.720)$, and resulted in a change in management in $36.95 \%$ of patients, 95\% CI $(0.303,0.435)$ (Figure 2).

\section{Discussion}

POC ultrasound is currently used in the management of many disease processes seen in the critically ill patient and is considered in many instances part of the standard of care for patients management. For many years, ICU physicians have been using POC ultrasound in their practice and research of the use of POC ultrasound has been increasing. Formal curriculum is yet to be standardized for training the ICU fellows. Many societies have announced support and published statements for the use of POC ultrasound $[29,30]$. The curriculum that we designed was adopted from other successful programs and after careful review of literature and years of experience. Most critical care programs that implement a curriculum use something similar in core teaching and training.

POC ultrasound research in the ICU has expanded greatly over the last 10 years. Most studies have focused on system based ultrasound exams or certain protocols [20, 23-25]. Many studies have evaluated the performance of POC ultrasound by nonradiologists in comparison to radiologists. This is the first study to report the outcomes from implementing a structured POC ultrasound curriculum in an ICU fellowship program.

Neri et al. [21] described the bedside ultrasound approach and implementing a curriculum in the ICU. Their approach was a systematic airway, breathing, circulation, deformity, and exposure (ABCDE) where all the systems were examined. The curriculum was extensive and required more time and training to achieve the required levels of competencies. Our curriculum addresses all the systems required to be examined with a focus on the presenting complaint and/or injury and critical problem-based pathways. It can serve as a more focused approach to the presenting problem, keeping in mind that at any time the operator can choose to perform a full exam as time permits. The curriculum we adopted was similar to the one endorsed by the ACEP/ASE [30] and is similar to the basic general competency (not advanced) presented by Neri.

Manno et al. [5] reported a study done in an ICU where patients were subjected to ICU-Sound Protocol. Findings showed that ultrasonographic findings modified the admitting diagnosis in $25.6 \%$, confirmed it in $58.4 \%$, were not effective in confirming or modifying it in $13.6 \%$, and missed it in $2.4 \%$ of patients. Manasia et al. [23] studied the applicability of echocardiography performed by intensivists after a 10-hour tutorial sessions and found a $94 \%$ success rate in performing the exams and documented that the limited echocardiographic exams performed by the intensivists lead to a change in management in $37 \%$ of cases. Lim et al. [17] studied the development of a multidisciplinary POC ultrasound curriculum and found in the needs assessment that all ICU fellows and faculty agreed on the need for formal training and only less than $50 \%$ were comfortable with the basics of the ultrasound machines and over $90 \%$ of both groups desired more training in the echocardiography discipline.

The learning curve for POC ultrasound is steep $[23,24]$. There are certain exams like the limited echocardiography exam that will require more time to master, but, generally, what we found was that implementing the curriculum with didactics and practice sessions for 30 hours followed by supervision of the trainees for a period of time, to achieve selfsufficient status, was achievable over a period of about 3 months.

Our study showed that implementing a structured POC ultrasound curriculum in the SICU fellowship program led 
to change in patient management with new diagnosis that would have been missed or delayed without the use of POC ultrasound. Our curriculum was designed on an existing ED model and modifications were done to adapt to our patient population. Many institutions have also adopted scanning protocols, which can be integrated in the curriculum.

The limitations of our study are that it was a single center, single ICU, retrospective data analysis with the possibility of bias being present since the POC ultrasound exam performer and interpreter were aware of the patient status and deciding the management path. Our results were not compared to the gold standard diagnostic modality for different diseases, but we relied on the opinion of expert staff members in POC ultrasound.

The curriculum we developed is not complete, and much more work and revisions will be needed. A structured curriculum needs to be conducted in the near future in a prospective manner with educational and patient outcomes measured. Those studies will help set the standard of care in POC ultrasound and help ICU physicians to use this modality in the correct and safe manner [22]. We are offering what we currently practice in our ICU in the hopes that this will stimulate more research and input from experts in the field. Following a structured curriculum with a rigorous QA process and follow-up is of utmost importance to the advancement in POC ultrasound and to our patient's care and safety $[22,31,32]$.

\section{Conclusions}

Implementation of POC ultrasound in the ICU with a structured fellowship curriculum was associated with an increase in new diagnosis in about $2 / 3$ and change in management in over $1 / 3$ of ICU patients studied.

\section{Conflict of Interests}

The authors declare that there is no conflict of interests regarding the publication of this paper.

\section{References}

[1] C. L. Moore and J. A. Copel, "Current concepts: point-of-care ultrasonography," The New England Journal of Medicine, vol. 364, no. 8, pp. 749-757, 2011.

[2] T. M. Scalea, A. Rodriguez, W. C. Chiu et al., "Focused assessment with sonography for trauma (FAST): results from an International Consensus Conference," Journal of Trauma-Injury, Infection and Critical Care, vol. 46, no. 3, pp. 466-472, 1999.

[3] A. W. Kirkpatrick, M. Sirois, K. B. Laupland et al., "Hand-held thoracic sonography for detecting post-traumatic pneumothoraces: the extended focused assessment with sonography for trauma (EFAST)," Journal of Trauma: Injury Infection \& Critical Care, vol. 57, no. 2, pp. 288-295, 2004.

[4] K. Killu, V. Coba, Y. Huang, T. Andrezejewski, and S. Dulchavsky, "Internal jugular vein collapsibility index associated with hypovolemia in the intensive care unit patients," Critical Ultrasound Journal, vol. 2, no. 1, pp. 13-17, 2010.
[5] E. Manno, M. Navarra, L. Faccio et al., "Deep impact of ultrasound in the intensive care unit: the "iCU-sound" protocol," Anesthesiology, vol. 117, no. 4, pp. 801-809, 2012.

[6] J. Elmer and V. E. Noble, "An evidence-based approach for integrating bedside ultrasound into routine practice in the assessment of undifferentiated shock," ICU Director, vol. 1, no. 3, pp. 163-174, 2010.

[7] M. D. Dana Sajed, "The history of point-of-care ultrasound use in disaster and mass casualty incidents," Virtual Mentor, vol. 12, no. 9, pp. 744-749, 2010.

[8] A. E. Sarkisian, R. A. Khondkarian, N. M. Amirbekian, N. B. Bagdasarian, R. L. Khojayan, and Y. T. Oganesian, "Sonographic screening of mass casualties for abdominal and renal injuries following the 1988 Armenian earthquake," Journal of Trauma: Injury Infection \& Critical Care, vol. 31, no. 2, pp. 247-250, 1991.

[9] M. Blaivas, D. Theodoro, and P. R. Sierzenski, "A study of bedside ocular ultrasonography in the emergency department," Academic Emergency Medicine, vol. 9, no. 8, pp. 791-799, 2002.

[10] A. B. Levitov, S. Aziz, and A. D. Slonim, "Before we go too far: ultrasound-guided central catheter placement," Critical Care Medicine, vol. 37, no. 8, pp. 2473-2474, 2009.

[11] M. Blaivas, L. Brannam, M. Hawkins, M. Lyon, and K. Sriram, "Bedside emergency ultrasonographic diagnosis of diaphragmatic rupture in blunt abdominal trauma," The American Journal of Emergency Medicine, vol. 22, no. 7, pp. 601-604, 2004.

[12] L. Chiao, S. Sharipov, A. E. Sargsyan et al., "Ocular examination for trauma; clinical ultrasound aboard the International Space Station," The Journal of Trauma, vol. 58, no. 5, pp. 885-889, 2005.

[13] A. E. Sargsyan, D. R. Hamilton, J. A. Jones et al., "FAST at MACH 20: clinical ultrasound aboard the International Space Station," Journal of Trauma-Injury, Infection and Critical Care, vol. 58, no. 1, pp. 35-39, 2005.

[14] S. Rao, L. van Holsbeeck, J. L. Musial et al., "A pilot study of comprehensive ultrasound education at the Wayne State University School of Medicine: a pioneer year review," Journal of Ultrasound in Medicine, vol. 27, no. 5, pp. 745-749, 2008.

[15] R. A. Hoppmann, V. V. Rao, M. B. Poston et al., "An integrated ultrasound curriculum (iUSC) for medical students: 4-year experience," Critical Ultrasound Journal, vol. 3, no. 1, pp. 1-12, 2011.

[16] S. M. Ismaeel, N. J. Day, D. Earnshaw, and J. W. Lorains, "Training requirements for point of care ultrasound in acute medicine," Acute Medicine, vol. 9, no. 2, pp. 87-90, 2010.

[17] S. Lim, A. Morris, M. Hallman, D. Sajed, and S. Hayden, "Development of a multidisciplinary point-of-care ultrasound curriculum for intensivists," American Journal of Respiratory and Critical Care Medicine, vol. 185, p. A5972, 2012.

[18] S. Sippel, K. Muruganandan, A. Levine, and S. Shah, "Review article: use of ultrasound in the developing world," International Journal of Emergency Medicine, vol. 4, article 72, 2011.

[19] D. J. van Hoving, H. H. Lamprecht, M. Stander et al., "Adequacy of the emergency point-of-care ultrasound core curriculum for the local burden of disease in South Africa," Emergency Medicine Journal, vol. 30, no. 4, pp. 312-315, 2013.

[20] R. Melamed, M. D. Sprenkle, V. K. Ulstad, C. A. Herzog, and J. W. Leatherman, "Assessment of left ventricular function by intensivists using hand-held echocardiography," Chest, vol. 135, no. 6, pp. 1416-1420, 2009.

[21] L. Neri, E. Storti, and D. Lichtenstein, "Toward an ultrasound curriculum for critical care medicine," Critical Care Medicine, vol. 35, no. 5, pp. S290-S304, 2007. 
[22] R. A. Filly, "Is it time for the sonoscope? If so, then let's do it right!," Journal of Ultrasound in Medicine, vol. 22, no. 4, pp. 323325, 2003.

[23] A. R. Manasia, H. M. Nagaraj, R. B. Kodali et al., "Feasibility and potential clinical utility of goal-directed transthoracic echocardiography performed by noncardiologist intensivists using a small hand-carried device (SonoHeart) in critically ill patients," Journal of Cardiothoracic and Vascular Anesthesia, vol. 19, no. 2, pp. 155-159, 2005.

[24] R. Razi, J. R. Estrada, J. Doll, and K. T. Spencer, "Bedside hand-carried ultrasound by internal medicine residents versus traditional clinical assessment for the identification of systolic dysfunction in patients admitted with decompensated heart failure," Journal of the American Society of Echocardiography, vol. 24, no. 12, pp. 1319-1324, 2011.

[25] G. Volpicelli, M. Elbarbary, M. Blaivas et al., "International evidence-based recommendations for point-of-care lung ultrasound," Intensive Care Medicine, vol. 38, no. 4, pp. 577-591, 2012.

[26] A. S. Graham, C. Ozment, K. Tegtmeyer, S. Lai, and D. A. V. Braner, "Central venous catheterization," The New England Journal of Medicine, vol. 356, article e21, 2007.

[27] D. C. McGee and M. K. Gould, "Preventing complications of central venous catheterization," The New England Journal of Medicine, vol. 348, no. 12, pp. 1123-1133, 2003.

[28] D. Seif, P. Perera, T. Mailhot, D. Riley, and D. Mandavia, "Bedside ultrasound in resuscitation and the rapid ultrasound in shock protocol," Critical Care Research and Practice, vol. 2012, Article ID 503254, 14 pages, 2012.

[29] P. H. Mayo, Y. Beaulieu, P. Doelken et al., "American college of chest physicians/ la societédé réanimation de langue française statement on competence in critical care ultrasonography," Chest, vol. 135, no. 4, pp. 1050-1060, 2009.

[30] A. J. Labovitz, V. E. Noble, M. Bierig et al., "Focused cardiac ultrasound in the emergent setting: a consensus statement of the American society of Echocardiography and American College of Emergency Physicians," Journal of the American Society of Echocardiography, vol. 23, no. 12, pp. 1225-1230, 2010.

[31] F. M. Abu-Zidan, "Point-of-care ultrasound in critically ill patients: where do we stand?" Journal of Emergencies, Trauma and Shock, vol. 5, no. 1, pp. 70-71, 2012.

[32] H. Edwards, "Let's all jump on the ultrasound bandwagon: further debate on the use of ultrasound," Ultrasound, vol. 18, no. 1, pp. 4-7, 2010. 


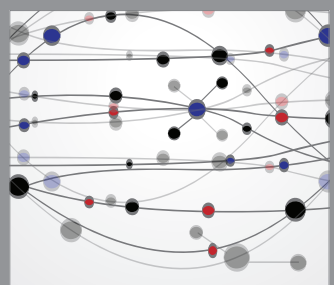

The Scientific World Journal
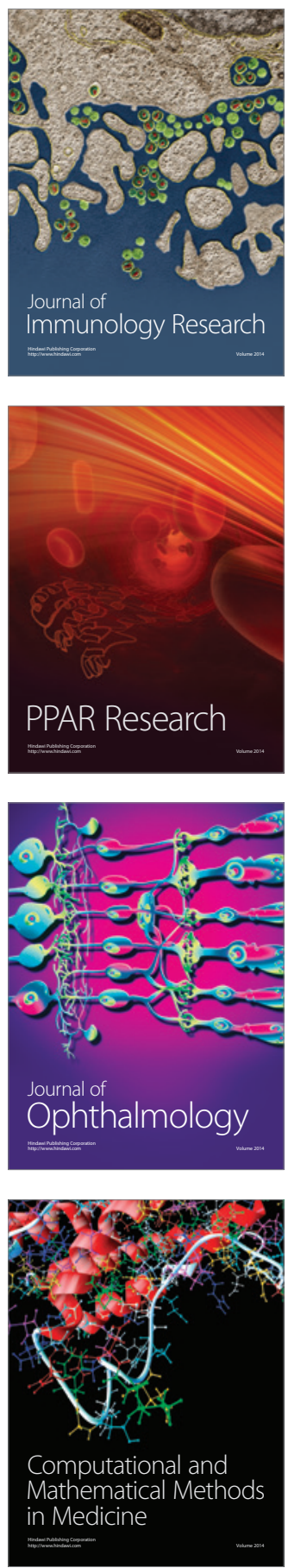

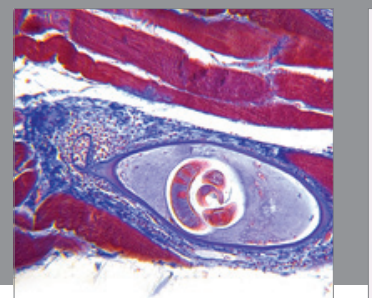

Gastroenterology

Research and Practice
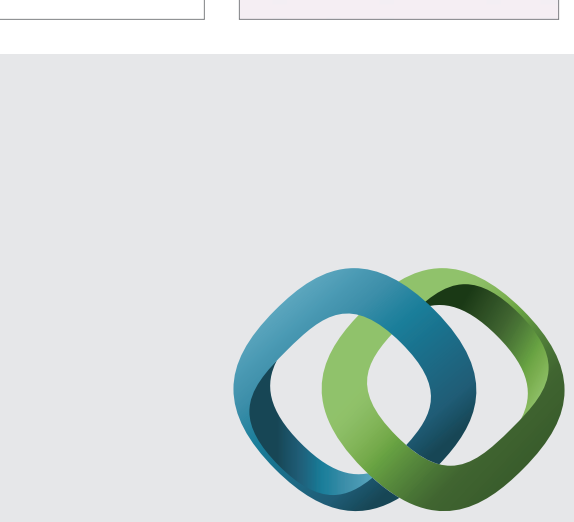

\section{Hindawi}

Submit your manuscripts at

http://www.hindawi.com
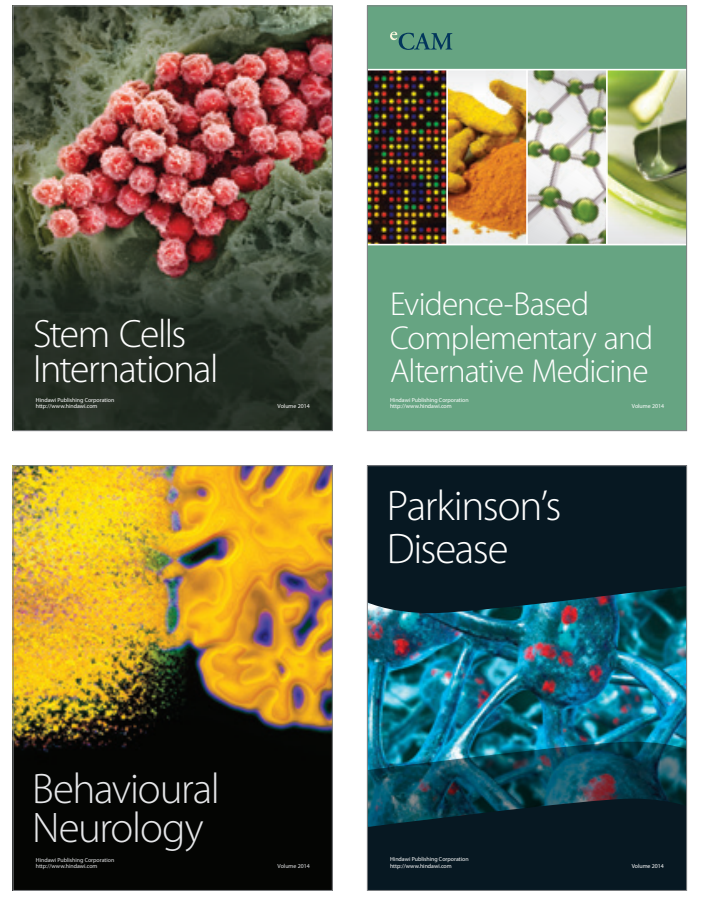
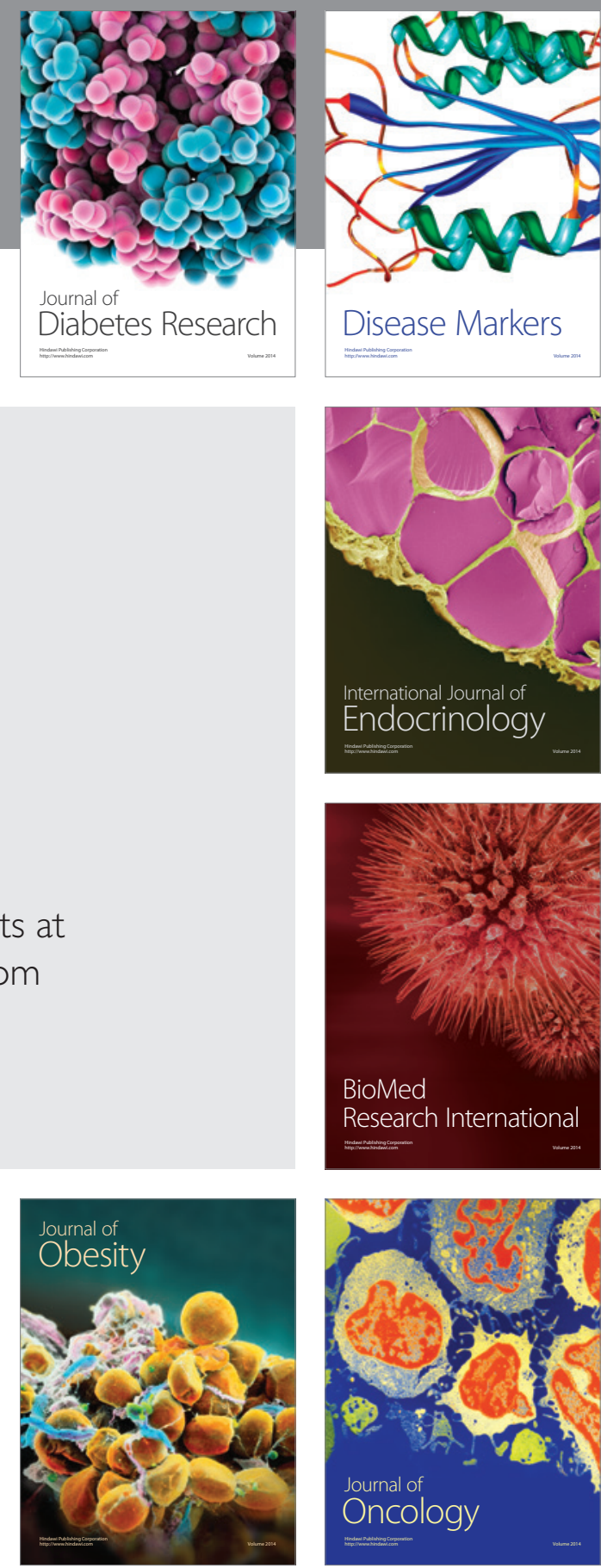

Disease Markers
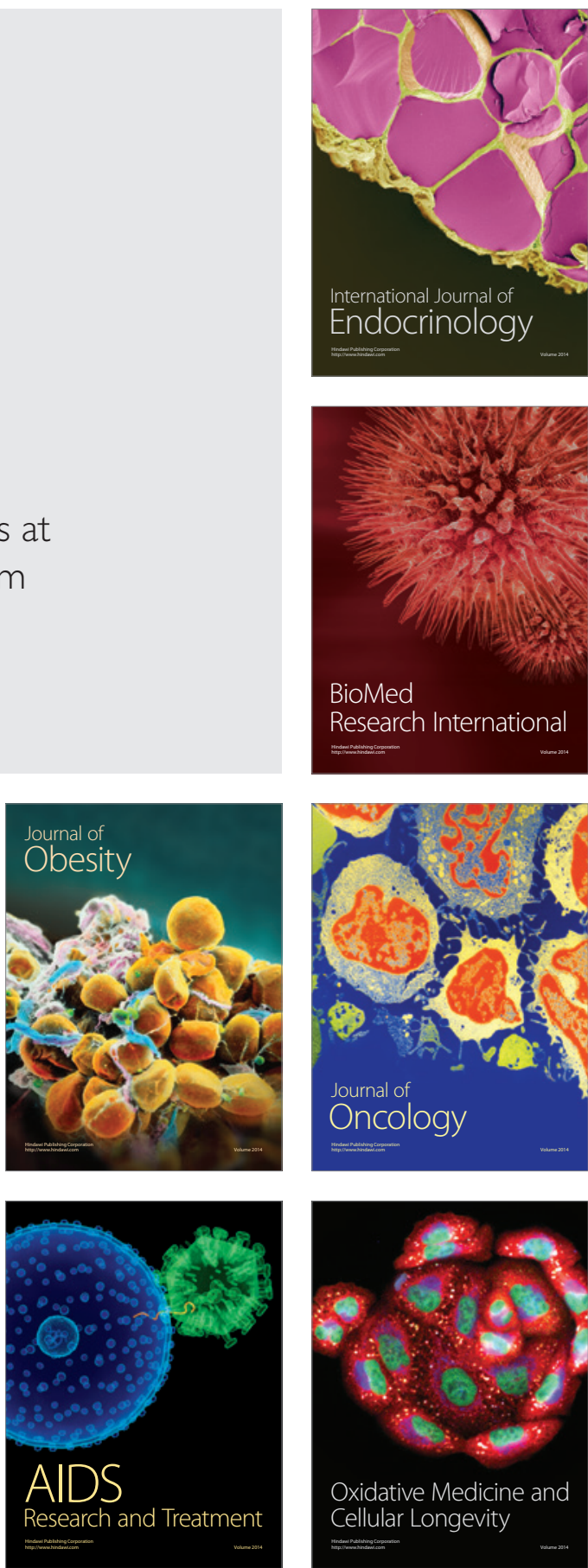